\title{
Antibody-Mediated Encephalitis
}

\author{
Josep Dalmau, M.D., Ph.D., and Francesc Graus, M.D., Ph.D.
}

From the Neurology Service, Hospital Clinic and Neuroimmunology Program, August $\mathrm{Pi}$ i Sunyer Biomedical Research Institute, University of Barcelona (J.D., F.G.), and the Catalan Institution for Research and Advanced Studies (J.D.) both in Barcelona; and the Department of Neurology, University of Pennsylvania, Philadelphia (J.D.). Address reprint requests to Dr. Dalmau at the Hospital Clinic, University of Barcelona, Casanova 143, Fl. 3a, Barcelona 08036, Spain, or at jdalmau@clinic.cat.

N Engl J Med 2018;378:840-51. DOI: $10.1056 /$ NEJMral708712

Copyright () 2018 Massachusetts Medical Society.

\section{A} NTIBODY-MEDIATED ENCEPHALITIDES CONSTITUTE A GROUP OF INflammatory brain diseases that are characterized by prominent neuropsychiatric symptoms and are associated with antibodies against neuronal cell-surface proteins, ion channels, or receptors (Table 1). ${ }^{1}$ Common clinical features include a change in behavior, psychosis, seizures, memory and cognitive deficits, abnormal movements, dysautonomia, and a decreased level of consciousness. There are, however, no systemic manifestations other than autonomic dysfunction, and this group of diseases is separable from traditional autoimmune disorders such as systemic lupus erythematosus, which may affect the nervous system. Also separate from this group of antibody-mediated encephalitides are several disorders, some of which are paraneoplastic, such as cerebellar degeneration, ${ }^{2}$ neuromyelitis optica, ${ }^{3}$ and stiff-person spectrum diseases, ${ }^{4}$ that are associated with antibodies against neuronal or glial cell-surface antigens but that are rarely associated with the aforementioned symptoms.

The antibody-mediated encephalitides occur in persons of all ages, with some types affecting predominantly children and young adults. Certain syndromes are recognizable on clinical grounds, and their autoimmune cause can be established with laboratory tests. Despite the severity of symptoms, prompt diagnosis and treatment lead to improvement or full recovery in most cases. This review focuses on the encephalitides associated with autoantibodies against neuronal cell-surface antigens, for which there is compelling evidence that the antibodies have direct pathogenic effects.

\section{FREQUENCY，IMMUNOLOGIC FEATURES, AND ASSOCIATED} DISORDERS

The estimated annual incidence of all types of encephalitis is approximately 5 to 8 cases per 100,000 persons, and in 40 to $50 \%$ of the cases, the cause cannot be established. ${ }^{5}$ A prospective, multicenter, population-based study suggests that autoimmune disorders are the third most common cause of encephalitis, after infections, usually viral, and acute disseminated encephalomyelitis, which is typically a postinfectious disorder. ${ }^{5} \mathrm{~A}$ study from a center that is specifically concerned with the epidemiology of encephalitis showed that the frequency of the most common form of autoimmune encephalitis, the type with antibodies against the N-methyl-Daspartate receptor (NMDAR), surpassed the frequency of any individual viral cause of encephalitis in young persons, ${ }^{6}$ and in one retrospective study, anti-NMDAR encephalitis accounted for $1 \%$ of all admissions of young adults to an intensive care unit. ${ }^{7}$ A retrospective Dutch study showed that encephalitis characterized by antibodies against leucine-rich, glioma-inactivated 1 (LGI1) was the second most frequent autoimmune encephalitis, with an incidence of 0.83 cases per 1 million persons. ${ }^{8}$ 


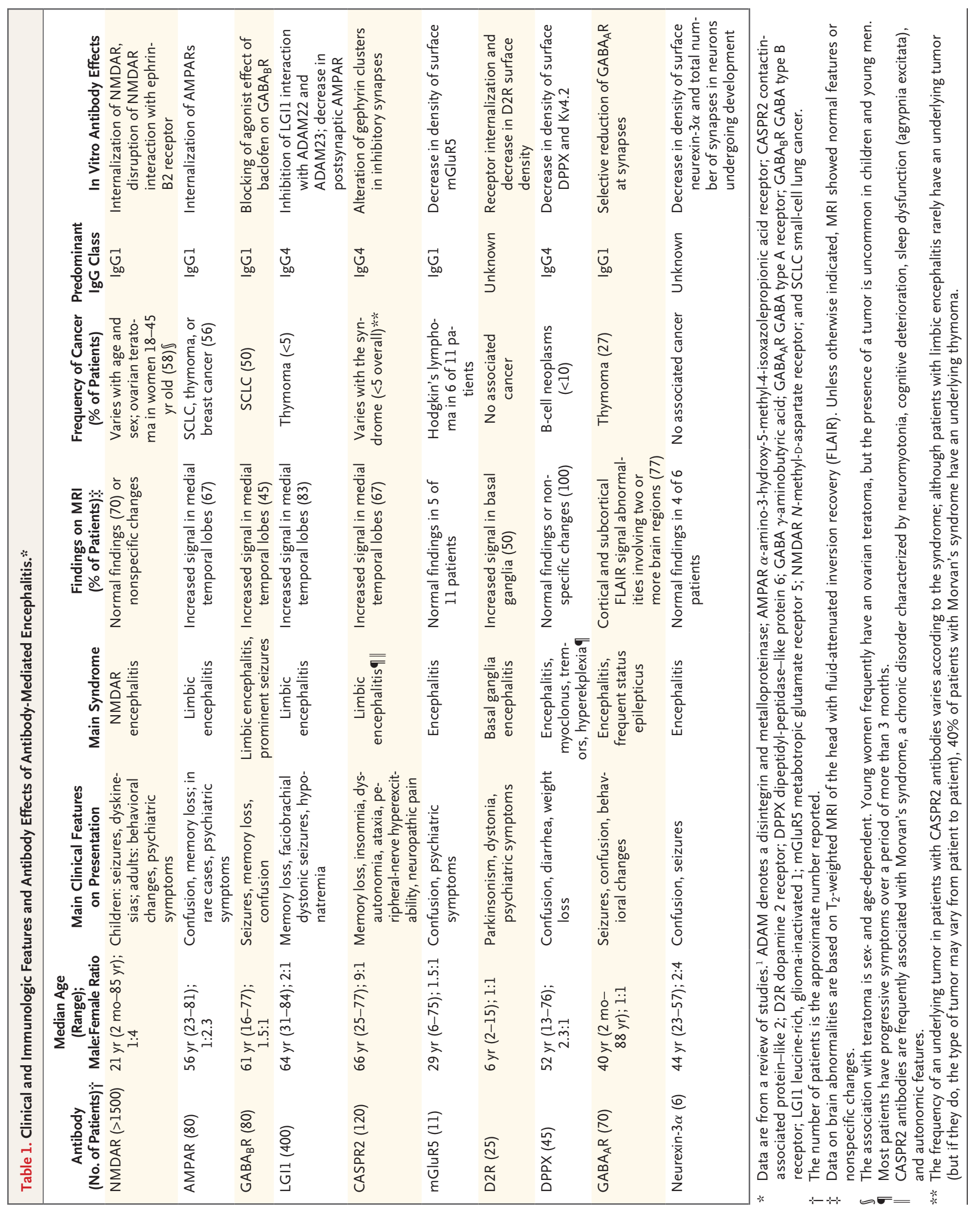


Beginning in the 1980s, studies of paraneoplastic neurologic syndromes associated with antibodies against intracellular neuronal antigens informed subsequent clinical and laboratory research on the autoimmune encephalitides. ${ }^{9}$ The distinction between these two groups of disorders is important because some of the triggers and syndromes are similar but their pathogenic mechanisms and outcomes are different. A comparison of the antibodies associated with these two categories is shown in Figure 1A through $1 \mathrm{~F}$. In the autoimmune encephalitides, the antibodies bind to extracellular epitopes of cell-surface proteins and cause reversible neuronal dysfunction. ${ }^{1}$ These features may explain the better outcomes for patients with autoimmune encephalitides, as compared with the outcomes for patients with neurologic syndromes related to antibodies against intracellular proteins, in which neuronal loss is frequent and cytotoxic T-cell mechanisms predominate ${ }^{10}$ (Fig. 1G through 1J).

Most autoimmune encephalitides occur in patients with no apparent immunologic triggers, leading some investigators to postulate a genetic predisposition to these disorders. Two studies showed an association of anti-LGI1 encephalitis with HLA class II genes, including HLA-DRB1 ${ }^{\star} 07$ (DR7) and HLA-DRB4 in a Dutch population ${ }^{11}$ and DRB1 ${ }^{\star} 07: 01-\mathrm{DQB1} 1^{\star} 02: 02$ in a Korean population. ${ }^{12}$ In the same two studies, no specific HLA association was found with anti-NMDAR encephalitis, ${ }^{12}$ but another study suggested a genetic predisposition in Maori and Pacific Island populations. ${ }^{13}$

Two potential triggers of autoimmune encephalitides are tumors (Table 1) and viral encephalitis. Some of the implicated tumors contain nerve tissue or the tumor cells express the neuronal proteins targeted by the autoantibodies, ${ }^{14}$ suggesting that the ectopic expression of these proteins may play a role in initiating the autoimmune response. Herpes simplex encephalitis, and possibly other viral encephalitides, can trigger antibodies against the NMDAR and other neuronal cell-surface proteins; such antibodies might explain relapsing neurologic symptoms that arise weeks after the onset of herpes simplex encephalitis. ${ }^{15,16}$ This delayed complication affects approximately $20 \%$ of patients with herpes simplex encephalitis and is manifested pre-
Figure 1 (facing page). Antibody Reactivity and Pathological Features of Encephalitis Associated with Antibodies against Neuronal Cell-Surface Antigens as Compared with Encephalitis Associated with Antibodies against Intracellular Antigens.

In encephalitis associated with antibodies against cellsurface antigens, the antibodies have access to the epitopes and can potentially alter the structure and function of the cognate antigen (Panel A), whereas in encephalitis associated with antibodies against intracellular antigens, the antibodies cannot reach the intracellular epitopes, and cytotoxic T-cell mechanisms are predominantly involved (Panel B). N-methyl-D-aspartate receptor (NMDAR) antibodies (Panels $\mathrm{C}$ and $\mathrm{E}$ ) are examples of the group of antibodies against cell-surface antigens, and $\mathrm{Hu}$ antibodies (Panels D and F) are examples of the group of antibodies against intracellular antigens. In immunofluorescence studies of rodent brain with tissue permeabilized to allow entry of antibodies, NMDAR antibodies are characterized by a pattern of neuropil-like immunolabeling (Panel C, green staining), whereas $\mathrm{Hu}$ antibodies have a discrete pattern of cellular immunolabeling (Panel D, green staining). In contrast, with live cultured neurons, NMDAR antibodies have access to the target antigen (Panel E, intensive immunolabeling), whereas $\mathrm{Hu}$ antibodies cannot reach the intracellular antigen (Panel F, no immunolabeling). Autopsy studies have shown that patients with anti-NMDAR encephalitis have moderate brain inflammatory infiltrates along with plasma cells (Panel G, cells stained brown with a CD138 antibody), deposits of IgG (Panel H, diffuse brown staining with an antihuman IgG antibody), and microglial proliferation (Panel $\mathrm{H}$ inset, microglial cells stained red with a CD68 antibody), without evidence of T-cell-mediated neuronal loss (not shown). In contrast, patients with anti-Hu paraneoplastic encephalitis have extensive neuronal loss and inflammatory infiltrates (not shown); the T cells are in direct contact with neurons (Panel I, arrows; hematoxylin and eosin), probably contributing to neuronal degeneration through perforin and granzyme mechanisms (Panel), arrow; granzyme B staining). All human tissue sections (Panels $G$ through J) were obtained from the hippocampus.

dominantly as choreoathetosis in children and as psychiatric and behavioral alterations in adults. ${ }^{15,17}$ Immunotherapy with glucocorticoids, plasma exchange, intravenous immune globulin, or rituximab is partially effective during relapse and does not appear to confer a predisposition to reactivation of the herpes simplex virus. ${ }^{18}$

\section{CLINICAL SYNDROMES}

In most cases of autoimmune encephalitis, the clinical presentation and findings on magnetic 


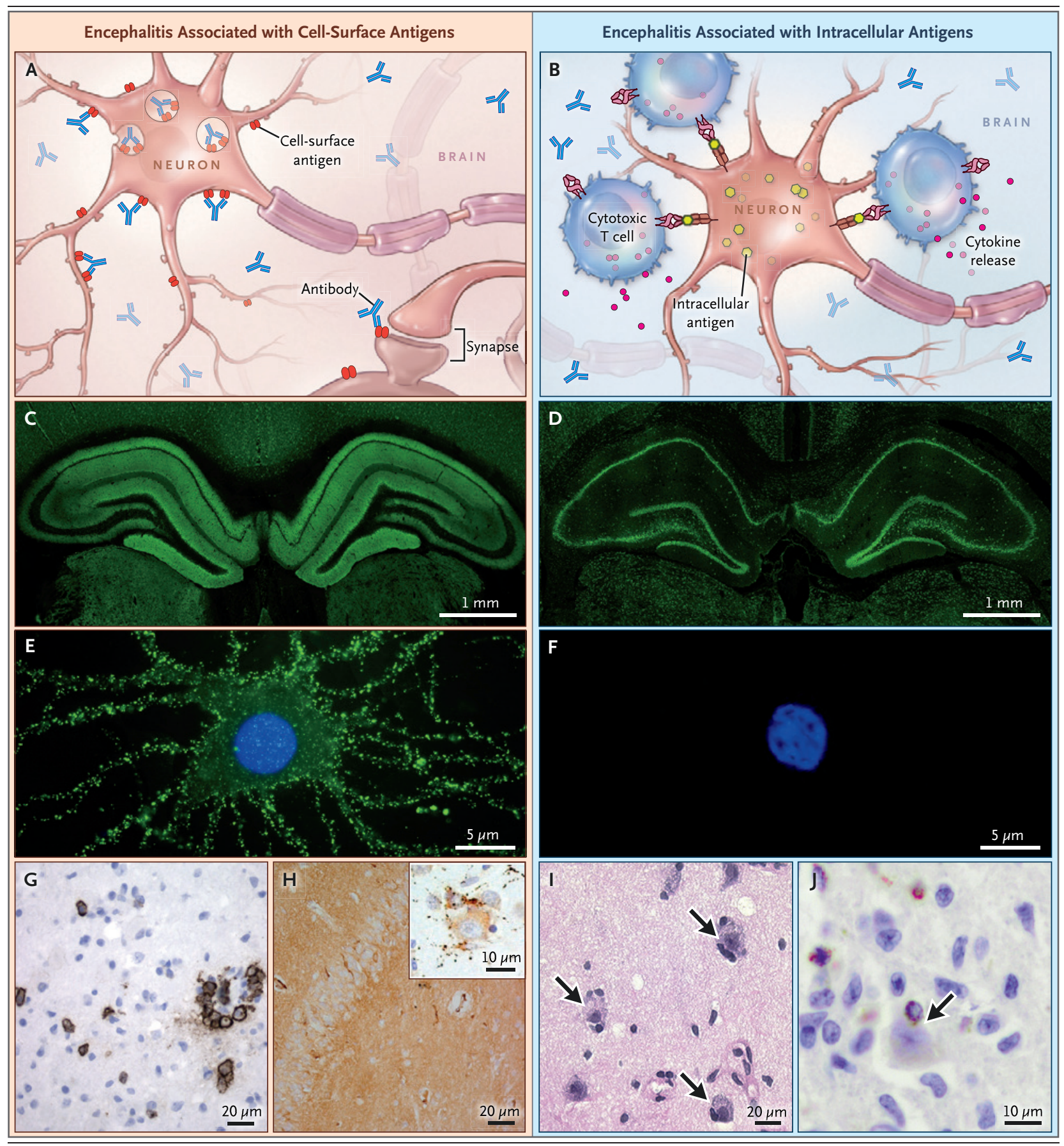

resonance imaging (MRI) of the head and cerebrospinal fluid (CSF) assessment resemble those in cases due to viral infection. ${ }^{19}$ Symptoms progress over a period of days or weeks, with the exception of some patients who have autoimmune encephalitis with antibodies against contactin- associated protein-like 2 (CASPR2), ${ }^{8}$ dipeptidylpeptidase-like protein 6 (DPPX), ${ }^{20}$ or LGI $1,{ }^{21}$ which may have a more indolent course. Approximately $60 \%$ of patients with autoimmune encephalitis have prodromal low-grade fever, malaise, or headache. Some prodromal symp- 
toms are characteristic of particular types of autoimmune encephalitides - for example, faciobrachial dystonic seizures and paroxysmal dizzy spells occur with anti-LGI1 encephalitis, ${ }^{22,23}$ and severe diarrhea and weight loss occur in the prodromal phase of anti-DPPX encephalitis ${ }^{24}$ (Table 1).

The disorders most frequently recognized on clinical grounds are anti-NMDAR encephalitis and limbic encephalitis. Anti-NMDAR encephalitis affects predominantly children and young adults (median age, 21 years), with a predominance of cases in females (4:1) that becomes less evident after the age of 45 years. ${ }^{25}$ Up to $58 \%$ of affected young female patients have an ovarian teratoma (extragonadal teratomas are a rare cause); in men and children, the association with tumors is less frequent. ${ }^{25}$ Young children typically present with insomnia, seizures, abnormal movements, or a change in behavior such as irritability, temper tantrums, agitation, and reduction of verbal output. Teenagers and adults more often present with psychiatric symptoms, including agitation, hallucinations, delusions, and catatonia, which may lead to hospital admission for psychosis. The disease progresses in a period of days or weeks to include reduction of speech, memory deficit, orofacial and limb dyskinesias, seizures, decreased level of consciousness, and autonomic instability manifested as excess salivation, hyperthermia, fluctuations of blood pressure, tachycardia, or central hypoventilation. ${ }^{26}$ Bradycardia and cardiac pauses are infrequent but require a temporary pacemaker in some patients. One month after disease onset, regardless of the symptoms at presentation, most children and adults have a syndrome that combines several of the above-mentioned symptoms; in approximately $5 \%$ of patients, the disease may remain monosymptomatic (e.g., psychiatric symptoms). ${ }^{25}$

MRI of the head is abnormal in $30 \%$ of affected patients, mainly showing increased fluidattenuated inversion recovery (FLAIR) signal involving the cortical, subcortical, or cerebellar regions (Fig. 2A). ${ }^{25}$ The diagnosis of anti-NMDAR encephalitis is confirmed by the detection of CSF antibodies against the GluN1 subunit of the NMDAR; serum testing is less reliable, with false negative results in up to $14 \%$ of cases. ${ }^{27}$ In children who have symptoms suggestive of anti-
NMDAR encephalitis but with discordant MRI changes involving the basal ganglia and brain stem, the possibility of encephalitis due to antibodies against the dopamine 2 receptor should be considered (Fig. 2B). ${ }^{28}$

In contrast to anti-NMDAR encephalitis, limbic encephalitis can result from immune responses against several different neuronal cellsurface proteins (Table 1). ${ }^{19}$ Patients with limbic encephalitis are usually older than 45 years, with a sex predominance that varies with the type of antibody (Table 1). Symptoms include confusion, behavioral changes, seizures, and inability to form new memories, with relative preservation of the old ones. The MRI scan shows increased FLAIR signal in the medial aspect of the temporal lobes, which in rare cases is enhanced with gadolinium infusion. In some cases, the MRI scan is normal or shows unilateral changes (Fig. 2C). If only one temporal lobe is involved, the differential diagnosis includes cortical edema from ongoing seizures, glioma, and herpes simplex encephalitis.

The likelihood and type of underlying tumor and the response to treatment differ according to the type of limbic encephalitis. LGI1 antibodies account for the majority of cases of limbic encephalitis, and hyponatremia is a feature of $65 \%$ of these cases; an underlying tumor is rare. ${ }^{21}$ Limbic encephalitis associated with antibodies against $\gamma$-aminobutyric acid (GABA) type $B$ receptor $\left(\mathrm{GABA}_{\mathrm{B}} \mathrm{R}\right)$ and that associated with antibodies against $\alpha$-amino-3-hydroxy5-methyl-4-isoxazolepropionic acid receptor (AMPAR) are the next most frequent types of limbic encephalitis; 50 to $60 \%$ of patients with limbic encephalitis due to one of these antibodies have cancer (Table 1). ${ }^{29,30}$ Limbic encephalitis can also be a manifestation of the aforementioned conventional paraneoplastic syndromes with antibodies against intracellular antigens (e.g., $\mathrm{Hu}$ and $\mathrm{Ma} 2)^{9}$ or the $65-\mathrm{kD}$ isoform of glutamic acid decarboxylase (GAD65). These syndromes usually respond less well to immunotherapy than do the autoimmune encephalitides. ${ }^{19}$

Other autoimmune encephalitides (Table 1) have less distinctive symptoms and MRI findings. Certain clinical features nevertheless suggest a specific type of autoimmune encephalitis, such as refractory status epilepticus with 

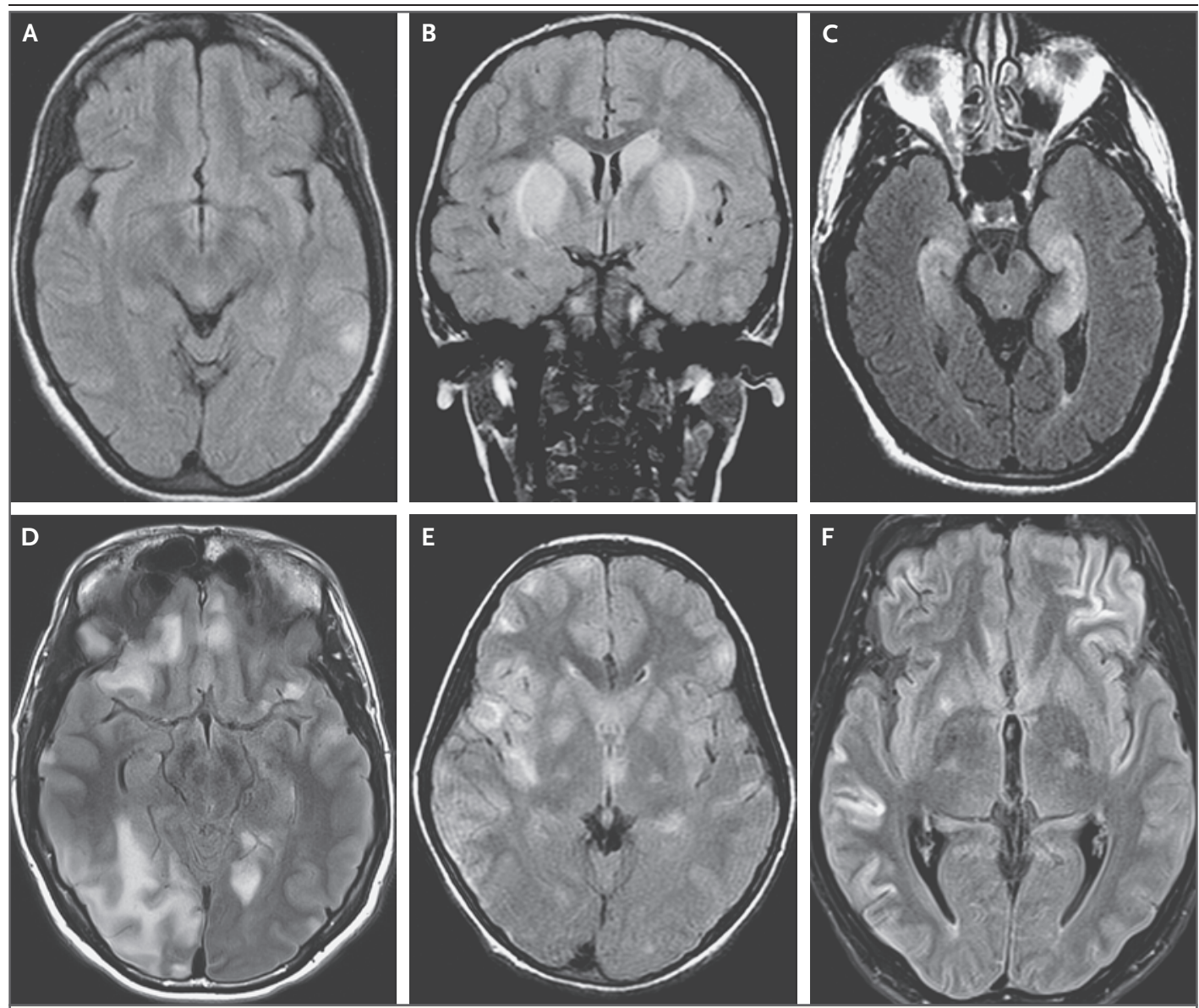

Figure 2. MRI Findings in Antibody-Mediated Encephalitis.

Shown are representative MRI scans from patients with several types of autoimmune encephalitides. Anti-NMDAR encephalitis often is present despite normal MRI findings or mild signal abnormalities on fluid-attenuated inversion recovery (FLAIR) images (Panel A). Basal ganglia encephalitis associated with dopamine 2 receptor antibodies typically affects the striatum (Panel B). Limbic encephalitis may result from several different immune responses and is typically indicated by FLAIR signal increases in the medial temporal lobes (Panel C). In contrast, encephalitis with antibodies against $\gamma$-aminobutyric acid $(G A B A)$ type $A$ receptor $\left(G A B A_{A} R\right)$ is usually associated with multiple cortical and subcortical FLAIR signal changes (Panel D). Patients with various acute inflammatory demyelinating diseases may have clinical and MRI findings that are indistinguishable from the findings in patients with autoimmune encephalitis. For example, an MRI scan showing extensive, bilateral FLAIR signal abnormalities was obtained from a patient in whom sudden-onset confusion and encephalopathy developed that were caused by acute disseminated encephalomyelitis associated with antibodies against myelin oligodendrocyte glycoprotein (Panel E). The clinical and radiologic features of autoimmune encephalitides can occasionally be misleading. For example, a young man was admitted for severe encephalitis and refractory seizures that required pharmacologically induced coma. Studies showed a large thymoma, $\alpha$-amino-3-hydroxy-5-methyl-4-isoxazolepropionic acid receptor (AMPAR) antibodies, and MRI findings (Panel F) that suggested widespread cortical damage and a poor prognosis. However, removal of the tumor and immunotherapy resulted in complete clinical recovery.

GABA type A receptor $\left(\mathrm{GABA}_{\mathrm{A}} \mathrm{R}\right)$ antibodies; encephalopathy, insomnia, dysautonomia, ataxia, peripheral-nerve hyperexcitability, and neuropathic pain with CASPR2 antibodies ${ }^{23,31}$; and myoclonus, tremors, and exaggerated star- tle responses (hyperekplexia) with DPPX antibodies. ${ }^{20,24}$

In most autoimmune encephalitides, the MRI is normal or shows nonspecific inflammatory changes; two exceptions are limbic encephalitis 
and anti-GABA $R$ encephalitis. In $\mathrm{GABA}_{\mathrm{A}} \mathrm{R}$ encephalitis, which occurs predominantly in children and young adults, FLAIR images show multifocal cortical and subcortical signal abnormalities, mainly in the frontal and temporal lobes and less frequently in the cerebellum and basal ganglia. ${ }^{32}$ These lesions do not show diffusion restriction or contrast enhancement and resemble the lesions in acute disseminated encephalomyelitis (Fig. 2D).

Most patients with autoimmune encephalitis have moderate CSF lymphocytic pleocytosis $(<100$ cells per cubic millimeter), but the absence of pleocytosis does not rule out the diagnosis. The encephalitides with LGI1 or DPPX antibodies are the ones that most frequently occur with normal MRI findings and normal results of standard CSF studies (cell count and protein and glucose levels). ${ }^{20,21}$ In most patients with autoimmune encephalitis, even those with normal findings on standard CSF studies, neuronal autoantibodies are detected in $\mathrm{CSF}^{19}$ However, some patients with anti-LGI1 encephalitis have antibodies that are detectable only in serum ${ }^{23}$ or only in CSF. ${ }^{21}$

The differential diagnosis of autoimmune encephalitis, which is extensive, ${ }^{19}$ includes acute disseminated encephalomyelitis, characterized by MRI abnormalities throughout white and gray matter and frequent detection of autoantibodies against myelin oligodendrocyte glycoprotein (Fig. 2E), and neuromyelitis optica spectrum disorders, in which the MRI abnormalities are often adjacent to periventricular and ependymal regions and most patients have antibodies against the water channel aquaporin-4, which is expressed in the endfeet of astrocytes. For unexplained reasons, these demyelinating syndromes can develop concurrently with anti-NMDAR encephalitis, resulting in overlapping clinical syndromes. ${ }^{33}$ The clinical and MRI features of autoimmune encephalitides can occasionally suggest a neurodegenerative process ${ }^{21}$ or irreversible brain damage reflected by restricted diffusion in regions of the cerebral cortex (Fig. 2F). Clinical recognition of these atypical presentations of autoimmune encephalitides is important because they are potentially treatable with immunotherapy.

\section{MECHANISMS OF DISEASE}

The target antigens in autoimmune encephalitides are cell-surface proteins involved in neuronal
Figure 3 (facing page). Proposed Mechanisms of Disease and Functional Interactions of Autoantibodies with Neuronal Surface Proteins.

A multistep process results in antibody-mediated neuronal cell dysfunction; some of the steps have been shown in reported studies, whereas others are based on proposed hypotheses. Two well-known triggers of autoimmune encephalitides are represented: herpes simplex virus (Panel A) and systemic tumors (Panel B). It is postulated that antigens released by virus-induced neuronal cell destruction or apoptotic tumor cells are loaded into antigen-presenting cells (dendritic cells) and transported to regional lymph nodes. ${ }^{9}$ In the lymph nodes, naive $B$ cells exposed to the processed antigens, in conjunction with CD4+ T cells, become antigenconditioned and differentiate into antibody-producing plasma cells. After entering the brain, memory B cells undergo restimulation, antigen-driven affinity maturation, clonal expansion, and differentiation into antibody producing plasma cells (Panel C). ${ }^{35}$ The contribution of systemically produced antibodies to the pool of antibodies present in the brain is unclear and may depend on systemic antibody titers and the integrity of the blood-brain barrier. On the basis of experimental models with cultured neurons, the presence of antibodies in the brain may lead to neuronal dysfunction through various mechanisms, including functional blocking of the target antigen (GABA type $B$ receptor $\left[G A B A_{B} R\right]$ antibodies, Panel D), receptor cross-linking and internalization (NMDAR antibodies, Panel E), and disruption of protein-protein interactions (leucine-rich, glioma-inactivated 1 [LGII]), potentially affecting the function of the voltage-gated potassium channels and leading to a decrease in the levels of AMPAR (Panel F). ${ }^{1}$ These mechanisms are influenced by the type of antibodies; for example, IgGl antibodies frequently crosslink and internalize the target antigen, but $\lg G 4$ antibodies are less effective in cross-linking the target and more often alter protein-protein interactions. A graph based on an in vivo model (Panel G) shows how antibodies from patients with anti-NMDAR encephalitis cause symptoms. In this mouse model, passive cerebroventricular infusion of antibodies during 14 days was associated with a progressive increase in brainbound human antibodies, which was maximal on day 18 . The antibodies caused a progressive decrease in synaptic NMDAR and loss of memory. All findings were reversed a few days after cessation of the antibody infusion, including a gradual decrease in levels of antibodies in the mouse hippocampus and restoration of the density of synaptic NMDAR and memory function. ${ }^{36}$

signaling and synaptic plasticity. ${ }^{1}$ The associated syndromes show substantial resemblance to the syndromes observed when the function of the same proteins is altered by genetic modification or pharmacologic antagonists. For example, many clinical features of anti-NMDAR encephalitis resemble those observed with the adminis- 


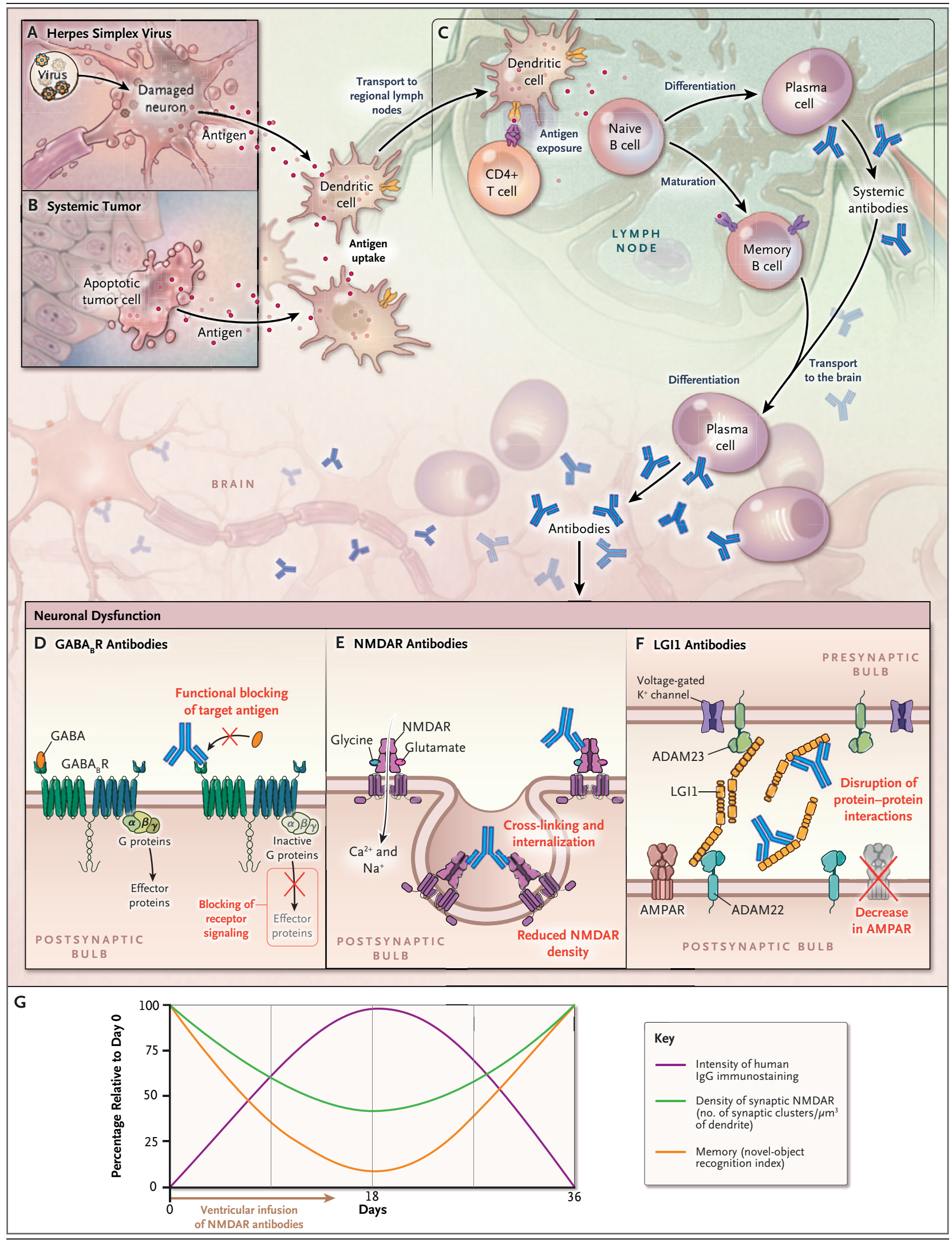

N ENGLJ MED 378;9 NEJM.ORG MARCH 1, 2018

The New England Journal of Medicine

Downloaded from nejm.org at MAHIDOL UNIVERSITY on March 4, 2018. For personal use only. No other uses without permission.

Copyright @ 2018 Massachusetts Medical Society. All rights reserved. 
tration of noncompetitive NMDAR antagonists (ketamine or phencyclidine). ${ }^{34}$ The ways in which the immune response is initiated and the antibodies reach or are produced in the brain are starting to be elucidated. It has been postulated that the autoimmune response is initiated by antigens released by the viral destruction of neurons (e.g., in herpes simplex encephalitis) (Fig. 3A), by tumors (Fig. 3B), or by unknown mechanisms. In the case of antiNMDAR encephalitis, there is preliminary evidence that memory $\mathrm{B}$ cells reach the brain, where they undergo restimulation, antigendriven affinity maturation, clonal expansion, and differentiation into antibody-producing plasma cells (Fig. 3C). This is supported by brain biopsy and autopsy studies showing plasma cells (Fig. 1G), deposits of IgG (Fig. 1H), and reduced levels of NMDAR ${ }^{37,38}$ and by CSF studies showing an ongoing, antigen-driven, intrathecal immune response characterized by clonally expanded plasma cells producing antibodies against NMDAR. ${ }^{35}$ Similar mechanisms may apply to those autoimmune encephalitides that are also characterized by intrathecal synthesis of antibodies, little clinical evidence of bloodbrain barrier disruption, and low or undetectable serum antibody levels in patients with severe deficits.

For all autoimmune encephalitides, pathogenic effects of the antibodies have been shown in primary cultures of neurons. These effects include blocking of receptor function (e.g., in the case of $\mathrm{GABA}_{\mathrm{B}} \mathrm{R}$ ), cross-linking and internalization of receptors (NMDAR), ${ }^{38,39}$ and interference with protein-protein interactions (LGI1) ${ }^{40}$ (Table 1 and Fig. 3D, 3E, and 3F). Even though some antibodies are of subclass IgG1 or IgG3, there is limited evidence that complement fixation plays a major role in autoimmune encephalitides. ${ }^{10,37}$ In a mouse model involving passive cerebroventricular transfer of antibodies from the CSF of affected patients ${ }^{36,41}$ or of a human recombinant antibody derived from CSF plasma cells, ${ }^{35}$ the antibodies disrupted the interaction between NMDAR and the ephrin-B2 receptor, leading to receptor internalization, impairment of long-term synaptic plasticity, memory deficits, anhedonia, and depressive behaviors. These alterations gradually resolved after the antibody infusion was stopped (Fig. 3G). ${ }^{36}$ The pathogenicity of NMDAR antibodies from affected patients has been suggested in other experimental models. ${ }^{42,43}$ No animal models are available for other autoimmune encephalitides.

\section{TREATMENTS AND OUTCOME}

Treatment recommendations are based largely on retrospective series and expert opinion, since few clinical trials have been conducted. The current approach includes immunotherapy and removal of the immunologic trigger, such as teratoma or another tumor, when applicable. Early tumor treatment is particularly important in achieving a good outcome. ${ }^{25,29}$ In most autoimmune encephalitides, antibody production and inflammatory changes occur behind the bloodbrain barrier, which probably explains the limited effectiveness of plasma exchange and of intravenous immune globulin, in contrast to the beneficial effects of these interventions in systemic antibody-mediated diseases such as myasthenia gravis. Nevertheless, in practice, most patients are treated with glucocorticoids, intravenous immune globulin, or plasma exchange, and if there is no clinical response, rituximab and cyclophosphamide are used. ${ }^{25}$ Rituximab is usually effective in refractory cases, and it appears to reduce the risk of a clinical relapse, ${ }^{25}$ which accounts for its increasing use as an initial treatment. ${ }^{44}$ Although hyperthermia, muscle rigidity, mutism, and coma may develop in patients with anti-NMDAR encephalitis independent of the use of neuroleptic agents, studies suggest an increased susceptibility to the adverse effects of these drugs (e.g., the neuroleptic malignant syndrome); the mechanisms underlying this complication are unknown. ${ }^{45}$

The speed of recovery, degree of residual deficit, and frequency of relapse vary according to the type of autoimmune encephalitis. In a series of 577 patients with anti-NMDAR encephalitis, 53\% had clinical improvement within 4 weeks, and $81 \%$ had substantial recovery (i.e., mild or no residual symptoms) at 24 months. ${ }^{25}$ Another study showed that patients with antiLGI1 encephalitis had a more rapid response but that only $70 \%$ had substantial recovery at 24 
months. ${ }^{21}$ For autoimmune encephalitides that are frequently associated with cancer, such as anti-AMPAR and anti-GABA $R$ encephalitides, the rate of response to immunotherapy is lower, particularly when additional paraneoplastic mechanisms such as antibodies and cytotoxic T-cell responses against intracellular antigens are identified. ${ }^{29}$

For all types of autoimmune encephalitides, prompt immunotherapy has been associated with a favorable outcome; spontaneous clinical improvement is infrequent. ${ }^{25}$ The frequency of clinical relapse in the encephalitides associated with antibodies against NMDAR, AMPAR, LGI1, CASPR2, or DPPX ranges from 12 to $35 \%$. $8,21,24,25,29$ Relapses often occur when immunotherapy is reduced or discontinued..$^{23}$ There is anecdotal evidence that cases of anti-LGI1 or anti-NMDAR encephalitis can relapse many years after the first episode. Relapses may herald recurrence of the associated tumor or a tumor that was missed in the initial episode. ${ }^{25}$ Immunotherapy and treatment of the tumor, if it was missed initially, usually result in improvement.

\section{FUTURE STUDIES}

The discovery of the category of autoimmune encephalitides has changed the diagnostic and treatment approach to many neurologic or psychiatric syndromes that were previously considered to be idiopathic. The rapid increase in the number of syndromes and autoantibodies identified over the past 10 years suggests that other autoimmune encephalitides have yet to be discovered. ${ }^{1}$ Antibody titers correlate imperfectly with the course of the disease and may remain detectable (albeit at a low titer) after clinical re- covery, ${ }^{27}$ indicating the need to identify biomarkers for prognosis and treatment decisions. The usefulness of neuropsychological testing, electroencephalography, ${ }^{46}$ advanced neuroimaging, ${ }^{47}$ and ${ }^{18} \mathrm{~F}$-fluorodeoxyglucose-positron-emission tomography ${ }^{48}$ in the diagnosis of autoimmune encephalitides, assessment of treatment efficacy, and prognosis requires investigation. Preliminary data suggest that the protracted clinical course of anti-NMDAR encephalitis is due to antibody production by long-lived plasma cells in the brain, ${ }^{35}$ along with the antibody effects on brain circuitry. ${ }^{41}$ Further studies are needed to confirm these hypotheses and determine whether they apply to other autoimmune encephalitides. Studies of how autoantibodies alter the structure and function of synaptic proteins and cause symptoms are critical for an understanding of the underlying pathogenic mechanisms, which in turn could lead to the development of new treatment strategies. For example, the observation that NMDAR antibodies alter the interaction between NMDAR and the ephrin-B2 receptor ${ }^{49}$ and that a soluble agonist of the ephrin-B2 receptor antagonizes the antibody effects suggests a potential treatment strategy. ${ }^{41}$ Finally, knowing how antibodies cause symptoms, such as the psychosis caused by anti-NMDAR antibodies, may help to understand psychiatric diseases in which the same receptors may be altered by other mechanisms. ${ }^{50}$

Disclosure forms provided by the authors are available with the full text of this article at NEJM.org.

We thank Drs. Russell Dale and Shekeeb Mohammad (Children's Hospital at Westmead, Australia) and Drs. Leslie Benson and Mark Gorman (Boston Children's Hospital) for providing MRI scans and Dr. Myrna R. Rosenfeld for reviewing an earlier version of the manuscript.
REFERENCES

1. Dalmau J, Geis C, Graus F. Autoantibodies to synaptic receptors and neuronal cell surface proteins in autoimmune diseases of the central nervous system. Physiol Rev 2017;97:839-87.

2. de Graaff E, Maat P, Hulsenboom E, et al. Identification of delta/notch-like epidermal growth factor-related receptor as the $\mathrm{Tr}$ antigen in paraneoplastic cerebellar degeneration. Ann Neurol 2012;71: 815-24.

3. Hinson SR, Romero MF, Popescu BF, et al. Molecular outcomes of neuromyelitis optica (NMO)-IgG binding to aquaporin-4 in astrocytes. Proc Natl Acad Sci U S A 2012;109:1245-50.

4. Carvajal-González A, Leite MI, Waters $\mathrm{P}$, et al. Glycine receptor antibodies in PERM and related syndromes: characteristics, clinical features and outcomes. Brain 2014;137:2178-92.

5. Granerod J, Ambrose HE, Davies NW, et al. Causes of encephalitis and differences in their clinical presentations in
England: a multicentre, population-based prospective study. Lancet Infect Dis 2010; 10:835-44.

6. Gable MS, Sheriff H, Dalmau J, Tilley $\mathrm{DH}$, Glaser CA. The frequency of autoimmune N-methyl-D-aspartate receptor encephalitis surpasses that of individual viral etiologies in young individuals enrolled in the California Encephalitis Project. Clin Infect Dis 2012;54:899904.

7. Prüss H, Dalmau J, Harms L, et al. 
Retrospective analysis of NMDA receptor antibodies in encephalitis of unknown origin. Neurology 2010;75:1735-9.

8. van Sonderen A, Petit-Pedrol M, Dalmau J, Titulaer MJ. The value of LGI1, Caspr2 and voltage-gated potassium channel antibodies in encephalitis. Nat Rev Neurol 2017;13:290-301.

9. Darnell RB, Posner JB. Paraneoplastic syndromes involving the nervous system. N Engl J Med 2003;349:1543-54.

10. Bien CG, Vincent A, Barnett MH, et al. Immunopathology of autoantibody-associated encephalitides: clues for pathogenesis. Brain 2012;135:1622-38.

11. van Sonderen A, Roelen DL, Stoop JA, et al. Anti-LGI1 encephalitis is strongly associated with HLA-DR7 and HLA-DRB4. Ann Neurol 2017;81:193-8.

12. Kim TJ, Lee ST, Moon J, et al. AntiLGI1 encephalitis is associated with unique HLA subtypes. Ann Neurol 2017; 81:183-92.

13. Jones HF, Mohammad SS, Reed PW, et al. Anti-N-methyl-D-aspartate receptor encephalitis in $\mathrm{M}$ ori and Pacific Island children in New Zealand. Dev Med Child Neurol 2017;59:719-24.

14. Lancaster E, Lai M, Peng $X$, et al. Antibodies to the GABA(B) receptor in limbic encephalitis with seizures: case series and characterisation of the antigen. Lancet Neurol 2010;9:67-76.

15. Armangue T, Moris G, CantarínExtremera V, et al. Autoimmune postherpes simplex encephalitis of adults and teenagers. Neurology 2015;85:173643.

16. Linnoila JJ, Binnicker MJ, Majed M, Klein CJ, McKeon A. CSF herpes virus and autoantibody profiles in the evaluation of encephalitis. Neurol Neuroimmunol Neuroinflamm 2016;3(4):e245.

17. Hacohen Y, Deiva K, Pettingill P, et al. $\mathrm{N}$-methyl-D-aspartate receptor antibodies in post-herpes simplex virus encephalitis neurological relapse. Mov Disord 2014;29: 90-6.

18. Nosadini M, Mohammad SS, Corazza F, et al. Herpes simplex virus-induced anti-N-methyl-D-aspartate receptor encephalitis: a systematic literature review with analysis of 43 cases. Dev Med Child Neurol 2017;59:796-805.

19. Graus F, Titulaer MJ, Balu R, et al. A clinical approach to diagnosis of autoimmune encephalitis. Lancet Neurol 2016; 15:391-404.

20. Tobin WO, Lennon VA, Komorowski L, et al. DPPX potassium channel antibody: frequency, clinical accompaniments, and outcomes in 20 patients. Neurology 2014; 83:1797-803.

21. Ariño H, Armangué T, Petit-Pedrol
$M$, et al. Anti-LGI1-associated cognitive impairment: presentation and long-term outcome. Neurology 2016;87:759-65.

22. Irani SR, Stagg CJ, Schott JM, et al Faciobrachial dystonic seizures: the influence of immunotherapy on seizure control and prevention of cognitive impairment in a broadening phenotype. Brain 2013;136:3151-62.

23. Gadoth A, Pittock SJ, Dubey D, et al. Expanded phenotypes and outcomes among 256 LGI1/CASPR2-IgG-positive patients. Ann Neurol 2017;82:79-92.

24. Hara $M$, Ariño $H$, Petit-Pedrol $M$, et al. DPPX antibody-associated encephalitis: main syndrome and antibody effects. Neurology 2017;88:1340-8.

25. Titulaer MJ, McCracken L, Gabilondo I, et al. Treatment and prognostic factors for long-term outcome in patients with anti-NMDA receptor encephalitis: an observational cohort study. Lancet Neurol 2013;12:157-65.

26. de Montmollin E, Demeret S, Brulé N, et al. Anti-N-methyl-D-aspartate receptor encephalitis in adult patients requiring intensive care. Am J Respir Crit Care Med 2017;195:491-9.

27. Gresa-Arribas N, Titulaer MJ, Torrents $\mathrm{A}$, et al. Antibody titres at diagnosis and during follow-up of anti-NMDA receptor encephalitis: a retrospective study. Lancet Neurol 2014;13:167-77.

28. Dale RC, Merheb V, Pillai S, et al. Antibodies to surface dopamine-2 receptor in autoimmune movement and psychiatric disorders. Brain 2012;135:3453-68.

29. Höftberger R, van Sonderen A, Leypoldt $\mathrm{F}$, et al. Encephalitis and AMPA receptor antibodies: novel findings in a case series of 22 patients. Neurology 2015;84:2403-12.

30. Jeffery OJ, Lennon VA, Pittock SJ, Gregory JK, Britton JW, McKeon A. GABAB receptor autoantibody frequency in service serologic evaluation. Neurology 2013; 81:882-7.

31. Joubert B, Saint-Martin M, Noraz N, et al. Characterization of a subtype of autoimmune encephalitis with anti-contactin-associated protein-like 2 antibodies in the cerebrospinal fluid, prominent limbic symptoms, and seizures. JAMA Neurol 2016;73:1115-24.

32. Spatola M, Petit-Pedrol M, Simabukuro $\mathrm{MM}$, et al. Investigations in GABAA receptor antibody-associated encephalitis. Neurology 2017;88:1012-20.

33. Titulaer MJ, Höftberger R, Iizuka $T$, et al. Overlapping demyelinating syndromes and anti-N-methyl-D-aspartate receptor encephalitis. Ann Neurol 2014; 75:411-28.

34. Krystal JH, Karper LP, Seibyl JP, et al.
Subanesthetic effects of the noncompetitive NMDA antagonist, ketamine, in humans: psychotomimetic, perceptual, cognitive, and neuroendocrine responses. Arch Gen Psychiatry 1994;51:199-214. 35. Malviya M, Barman S, Golombeck KS, et al. NMDAR encephalitis: passive transfer from man to mouse by a recombinant antibody. Ann Clin Transl Neurol 2017;4: 768-83.

36. Planagumà J, Leypoldt $\mathrm{F}$, Mannara $\mathrm{F}$, et al. Human N-methyl D-aspartate receptor antibodies alter memory and behaviour in mice. Brain 2015;138:94-109.

37. Martinez-Hernandez E, Horvath J, Shiloh-Malawsky Y, Sangha N, MartinezLage M, Dalmau J. Analysis of complement and plasma cells in the brain of patients with anti-NMDAR encephalitis. Neurology 2011;77:589-93.

38. Hughes EG, Peng X, Gleichman AJ, et al. Cellular and synaptic mechanisms of antiNMDA receptor encephalitis. J Neurosci 2010;30:5866-75.

39. Kreye J, Wenke NK, Chayka M, et al. Human cerebrospinal fluid monoclonal $\mathrm{N}$-methyl-D-aspartate receptor autoantibodies are sufficient for encephalitis pathogenesis. Brain 2016;139:2641-52.

40. Ohkawa T, Fukata Y, Yamasaki M, et al. Autoantibodies to epilepsy-related LGI1 in limbic encephalitis neutralize LGI1ADAM22 interaction and reduce synaptic AMPA receptors. J Neurosci 2013;33:1816174.

41. Planagumà J, Haselmann $\mathrm{H}$, Mannara F, et al. Ephrin-B2 prevents N-methyl-Daspartate receptor antibody effects on memory and neuroplasticity. Ann Neurol 2016;80:388-400.

42. Li Y, Tanaka K, Wang L, Ishigaki Y, Kato N. Induction of memory deficit in mice with chronic exposure to cerebrospinal fluid from patients with anti-Nmethyl-D-aspartate receptor encephalitis. Tohoku J Exp Med 2015;237:329-38.

43. Wright S, Hashemi K, Stasiak L, et al. Epileptogenic effects of NMDAR antibodies in a passive transfer mouse model. Brain 2015;138:3159-67.

44. Nosadini M, Mohammad SS, Ramanathan S, Brilot F, Dale RC. Immune therapy in autoimmune encephalitis: a systematic review. Expert Rev Neurother 2015;15:1391-419.

45. Lejuste F, Thomas L, Picard G, et al. Neuroleptic intolerance in patients with anti-NMDAR encephalitis. Neurol Neuroimmunol Neuroinflamm 2016;3(5):e280. 46. Mohammad SS, Soe SM, Pillai SC, et al. Etiological associations and outcome predictors of acute electroencephalography in childhood encephalitis. Clin Neurophysiol 2016;127:3217-24. 
47. Heine J, Prüss $\mathrm{H}$, Bartsch T, Ploner CJ, Paul F, Finke C. Imaging of autoimmune encephalitis - relevance for clinical practice and hippocampal function. Neuroscience 2015;309:68-83.

48. Probasco JC, Solnes L, Nalluri A, et al. Abnormal brain metabolism on FDG-
PET/CT is a common early finding in NMDA encephalitis. Brain 2012;135:1606autoimmune encephalitis. Neurol Neu- 21.

roimmunol Neuroinflamm 2017;4(4): $\quad$ 50. Weickert CS, Fung SJ, Catts VS, et al. e352. 49. Mikasova L, De Rossi P, Bouchet D, tate receptor hypofunction in schizophreet al. Disrupted surface cross-talk between nia. Mol Psychiatry 2013;18:1185-92.

NMDA and Ephrin-B2 receptors in anti- Copyright (C) 2018 Massachusetts Medical Society. 